\title{
New Insights in Resistant Diabetic Macular Edema
}

\author{
Dante Akira Kondo Kuroiwa Fernando Korn Malerbi \\ Caio Vinicius Saito Regatieri \\ Department of Ophthalmology and Visual Science, Federal University of São Paulo, São Paulo, Brazil
}

\section{Keywords}

Diabetic macular edema $\cdot$ Hard exudates $\cdot$ Blood-retinal barrier - Inflammation · Antivascular endothelial growth factor

\begin{abstract}
Diabetic macular edema (DME) is the most common cause of vision loss in diabetic eyes, and due to the rapid rise in the number of diabetic patients, the treatment burden has increased exponentially. The introduction of antivascular endothelial growth factor (anti-VEGF) therapy has been a major breakthrough in the management of center-involving DME, replacing laser photocoagulation as the first-line treatment. Despite the improvement in DME treatment with anti-VEGF therapy, persistent DME remains a challenge due to the extremely complex pathogenesis and the involvement of several different biochemical pathways. This review focuses on therapeutic options for persistent DME, which include corticosteroids, laser, and surgery. Novel agents for DME control such as new anti-VEGF, interleukin inhibitor, Rho-kinase inhibitor, and neuroprotective agents that are being investigated are reviewed as well. Future treatment perspectives include an individualized DME management.

(c) 2021 The Author(s)

Published by S. Karger AG, Basel
\end{abstract}

karger@karger.com www.karger.com/oph

Karger $\stackrel{\text { ' }}{5}$

GOPEN ACCESS
(C) 2021 The Author(s)

Published by S. Karger AG, Basel

This is an Open Access article licensed under the Creative Commons Attribution-NonCommercial-4.0 International License (CC BY-NC) (http://www.karger.com/Services/OpenAccessLicense), applicable to the online version of the article only. Usage and distribution for commercial purposes requires written permission.

\section{Introduction}

Diabetes mellitus (DM) is a global epidemic of the 21 st century. Currently, there are 382 million individuals with diabetes in the world, and this number is projected to reach 592 million by the year 2035 [1]. Diabetic macular edema (DME) is the most common cause of vision loss in diabetic eyes, and due to the rapid rise in the number of individuals with diabetes, the treatment burden has increased exponentially $[2,3]$.

The introduction of antivascular endothelial growth factor (anti-VEGF) therapy for DME has been a major breakthrough in the management of center-involving (CI)-DME, replacing laser photocoagulation as the firstline care. However, clinical response to anti-VEGF therapy may be highly variable, and the prevalence of persistent DME is estimated to be up to $50 \%[3,4]$. Other approaches such as therapy with intravitreal corticosteroids, subthreshold laser, and surgery have been considered in DME treatment [1].

DME pathogenesis is multifactorial, but increased oxidative stress, inflammation, vascular dysfunction, and neurodegeneration have been proposed as contributors to the development of DME. Persistent DME remains a challenge in the clinical practice, as it leads to chronic tissue stress and permanent disruption of the retinal architecture, causing photoreceptor loss $[4,5]$.

Correspondence to:

Dante Akira Kondo Kuroiwa, akira.epm78@gmail.com 
In this review, we describe the epidemiology and pathogenesis of DME, we define patterns of response to treatment, and we report novel treatment agents for persistent DME that target different biochemical pathways. We highlight the importance of individualizing the treatment for each patient. Data from preclinical and early clinical trials have been considered, and further trials will provide evidence for these agents in the future.

\section{Epidemiology}

The global prevalence of DM was 366 million in 2011, and it is expected to rise to 522 million by 2030 [4]. In recent decades, large increases in DM prevalence have been demonstrated in virtually all world regions [6]. DM describes a group of metabolic disorders characterized by increased blood glucose concentration [7] that leads to macrovascular complications, including coronary heart disease and stroke, and microvascular complications, such as end-stage renal disease and diabetic retinopathy (DR), responsible for much of the burden associated with DM [6].

DR affects approximately one-third of adults with DM and remains the leading cause of acquired vision loss worldwide in middle-aged, therefore, economically active people $[6,7]$. According to the $\mathrm{WHO}$, it is estimated that DR accounts for $4.8 \%$ of the number of cases of blindness [8]. The prevalence of DR in type $1 \mathrm{DM}$ was reported to range from 10 to $50 \%$. The overall prevalence of DR in type $2 \mathrm{DM}$ was $25.2 \%$ [8].

Among the visually disabling conditions in patients with diabetic eye disease, DME, left untreated, is a common cause of vision loss [9]. Among the populationbased studies, prevalence of DME among patients with type $1 \mathrm{DM}$ was between 4.2 and $7.9 \%$. In patients with type $2 \mathrm{DM}$, it was between 1.4 and $12.8 \%$ [10]. Durations of diabetes, hyperglycemia, and systemic hypertension are known risk factors for DR and DME [10].

\section{Pathogenesis}

DME is a multifactorial condition, and it involves thickening of the macula due to leakage of fluid from retinal capillaries caused by loss of pericytes, thickening of the basement membrane, and loss of tight junctions of the retinal endothelium from chronic hyperglycemia [4]. Multiple biochemical pathways play an important role in the pathophysiology of DME [4]. These include upregulation of VEGF, placental growth factor (PGF), angiopoi- etin-2 (Ang-2), intercellular adhesion molecule (ICAM1), interleukins, pigment epithelium-derived factor, matrix metalloproteinases, prostaglandins, and other cytokines [4].

Increased VEGF has emerged as one of the key factors in DME development, especially in early stages of the disease [11]. Activation of VEGF induces retinal vascular permeability by direct effect on endothelial tight junctions and adherence junctions [11]. With persistent DME, chronic inflammation assumes a more important role and propagates the edema [12]. Inflammatory mediators such as monocyte chemoattractant protein-1, tumor necrosis factor-alpha, interleukin-6 (IL-6), and interleukin-8 (IL-8) become more relevant in chronic DME [12]. Many studies have shown that the aqueous humor of DME patients contains elevated levels of inflammatory cytokines [12]. Some authors have described the association of aqueous IL- 8 , a well-known proinflammatory cytokine that acts as a neutrophil chemoattractant and a T-cell activator, and the responsiveness to intravitreal bevacizumab [12].

Although DME is considered a primarily vascular phenomenon with alteration of the blood-retinal barrier, recent works suggest that the pathology lies in alteration of the neurovascular unit that consists of Müller cells, astrocytes, ganglion cells, amacrine cells, retinal vascular endothelial cells, and pericytes [1]. The intimate dynamic interaction of retinal neurons and glia that surround the retinal capillaries controls fluid transport and metabolite transfer in the neural tissue, and abnormalities in these cells in DM affect this barrier property in the retinal vessels [1]. DME pathogenesis is extremely complex, and it is important to identify each contributing component to plan its management prudently [4].

\section{Classification and Diagnosis}

DME can occur in any stage of DR, either nonproliferative or proliferative retinopathy [1]. The Early Treatment Diabetic Retinopathy Study (ETDRS) defined "clinically significant macular edema" as (1) thickening of the retina within $500 \mu \mathrm{m}$ of the center of the macula; (2) hard exudates at or within $500 \mu \mathrm{m}$ of the center of the macula, if associated with thickening of the adjacent retina; or (3) a zone of retinal thickening $\geq 1$ disc area, any part of which lies within 1 disc diameter of the center of the macula $[13,14]$.

Although the clinical exam is still the gold standard for diagnosis of DME, optical coherence tomography (OCT) has now become a fast and convenient diagnostic tool for quantitative measurement and mapping of macular 
thickening [1]. Currently, diabetic eyes are generally classified by the International Council of Ophthalmology (ICO) as having no DME, noncentral-involved DME (retinal thickening in the macula that does not involve the central subfield zone, i.e., $1 \mathrm{~mm}$ in diameter), or centralinvolved DME (retinal thickening in the macula that does involve the central subfield zone, i.e., $1 \mathrm{~mm}$ in diameter).

\section{Response to Treatment}

Anti-VEGF agents have demonstrated remarkable efficacy in several pivotal clinical trials for control of CIDME and have become the gold standard primary treatment, replacing laser photocoagulation [4]. Differences between anti-VEGF drugs have been explored in Protocol $\mathrm{T}$, a comparative effectiveness trial conducted by the Diabetic Retinopathy Clinical Research Network (DRCR. net) that compared 3 commonly used anti-VEGF agents (bevacizumab, ranibizumab, and aflibercept) for eyes with CI-DME [15]. On average, each drug was found to improve vision and reduce central retinal thickness (CRT) [15]. Despite the efficacy of anti-VEGF agents in improving visual acuity and decreasing edema, approximately $40 \%$ of DME eyes experienced persistent DME after at least 6 monthly injections of anti-VEGF, and $32 \%$ of them had a concomitant visual loss $[5,15]$.

Characterization of responders to treatment of DME has been a challenge. A univocal definition of responsiveness to treatment has not been reached [15]. The DRCR. net proposed the definition of treatment as "success," "improvement," and "no improvement" after 6 monthly injections of anti-VEGF. "Success" was characterized by a bestcorrected visual acuity (BCVA) of 20/20 Snellen equivalent and CRT $<250 \mu \mathrm{m}$, "improvement" was defined by a visual gain of at least 5 letters and a decrease of CRT of at least $10 \%$, and "no improvement" included a BCVA change $<5$ letters and CRT $<10 \%$ since the baseline [15].

Persistent DME was defined by the DRCR.net as eyes with a CRT of $250 \mu \mathrm{m}$ or greater at 6 months of follow-up despite receipt of at least 4 of the potential 6 protocolmandated intravitreal anti-VEGFs during this period, and chronic persistent DME was defined as eyes with persistent DME that have not achieved a CRT $<250 \mu \mathrm{m}$ and $10 \%$ or greater reduction relative to a 6 -month follow-up visit on at least 2 consecutive study visits [16]. Recent studies suggested that eyes with persistent DME might benefit from a switch to dexamethasone implant early (only 3 anti-VEGF injections before switch) in the treatment course, providing a better visual and anatomical im- provement in unresponsive patients, when compared with late switch ( $>6$ anti-VEGF injections before dexamethasone) $[15,17]$.

\section{Biomarkers}

Intravitreal administration of anti-VEGF drugs is currently considered the first-line treatment for DME. However, not all patients with DME show a good response to anti-VEGF agents [18]. Approximately $32-66 \%$ of the eyes treated with intensive injection regimens over 6 months or beyond have persistent DME, and $30-70 \%$ of eyes show minimal improvement in BCVA [5].

In this context, many authors have described several biomarkers in DME to predict the response to anti-VEGF and corticosteroid therapy and to determine functional outcomes. Several authors have described OCT biomarkers that help to predict poorer visual outcome in patients with DME at the baseline, such as ellipsoid zone disruption, disorganization of the inner retinal layers in 1-mm foveal area, and lower parafoveal vascular density in the superficial layer on OCT angiography [19-21].

Some studies have suggested that the initial subfoveal choroidal thickness may help predict short-term antiVEGF treatment outcomes [22]. Rayess et al. [22] reported that patients having a greater baseline choroidal thickness were more likely to attain better visual and anatomic results with anti-VEGF therapy. Many authors have described OCT biomarkers that may represent an inflammatory pattern of DME [23]. The presence of subretinal fluid (SRF) and hyperreflective spots on OCT has been associated with major local inflammatory condition including higher levels of IL-6 in the vitreous [23]. Hyperreflective spots are described as small $(<30 \mu \mathrm{m})$, sometimes punctiform, reflective lesions, located in the outer retina, without any back shadowing, that may correspond to activated and aggregated microglial cells [23]. The presence of SRF has been described as a biomarker that predicts better visual outcome in patients treated with dexamethasone implant $[23,24]$.

Recently, aqueous humor cytokine levels have been used as biomarkers that could help anticipate response to anti-VEGF therapy [25]. Elevated ICAM-1 and reduced VEGF levels at baseline were associated with a favorable anatomic response to ranibizumab in DME [25]. Felfeli et al. [5] described that the VEGF concentration in aqueous humor was significantly lower in eyes with good response after 2 injections of aflibercept compared with eyes with a poor response to treatment. 


\section{First-Line Treatment for DME}

Decades after the Early Treatment Diabetic Retinopathy Study (ETDRS) established macular photocoagulation (focal/grid laser) as standard care for DME, antiVEGF injections have emerged as the first-line therapy for DME, with superior visual acuity outcomes and acceptable risks compared with focal/grid laser [26]. There is a large body of evidence to support the anti-VEGF use for DME. The RISE and RIDE studies showed that ranibizumab rapidly and sustainably improved vision and reduced macular thickness in patients with DME [27]. In the VISTA and VIVID trials, eyes treated with intravitreal aflibercept achieved significantly greater improvements in functional and anatomical outcomes when compared to macular laser photocoagulation [28].

The Protocol T from the DRCR.net has demonstrated that for eyes with a baseline BCVA of 20/30 to 20/40, no significant differences in visual outcomes are identified among aflibercept, bevacizumab, and ranibizumab [26, 28 ]. For eyes with a baseline BCVA of 20/50 or worse, aflibercept is associated with a superior visual acuity outcome up to 2 years of follow-up compared to bevacizumab, with a mean change in visual acuity of +18.1 letters score for the group treated with aflibercept and +13.3 letters score for the group treated with bevacizumab $[26,28]$. There was no significant difference between aflibercept and ranibizumab after 2 years in terms of visual acuity. Despite the minor difference in visual outcome, aflibercept is not cost-effective compared to bevacizumab $[26,28]$.

The combination of anti-VEGF with focal/grid photocoagulation could theoretically enhance the effect of laser photocoagulation because the retina would be less edematous if the laser treatment was administered sometime after the injection [29]. The Protocol I from the DRCR. net has demonstrated that intravitreal ranibizumab, either with prompt or deferred ( 24 weeks) focal/grid laser, resulted in superior visual acuity and OCT outcomes compared with focal/grid laser treatment without ranibizumab at both 1 and 2 years of follow-up [29].

\section{Options for Persistent DME}

\section{Switch of Anti-VEGF}

The rationale for switching between anti-VEGF drugs lies in the molecular differences and the pharmacological targets for each of these agents [4]. Bevacizumab, ranibizumab, and aflibercept each have different binding affinities for VEGF and different half-lives in the vitreous, and this could account for some of the differences noted between these drugs [30]. Ranibizumab is a small Fab fragment of the recombinant humanized monoclonal IgG1 antibody that binds and inhibits all isoforms of VEGF-A, and it lacks the Fc stem. Bevacizumab is a fulllength recombinant humanized monoclonal IgG1 antibody that binds and inhibits all isoforms of VEGF-A [30]. Aflibercept is a recombinant fusion protein that is composed of the extracellular binding domains from VEGF receptors 1 and 2, fused to the Fc domain of a human IgG1 molecule, and it binds and inhibits all isoforms of VEGF-A, but in addition also inhibits VEGF-B and PGF, unlike ranibizumab and bevacizumab $[4,31]$. Numerous authors have demonstrated that patients treated with repetitive ranibizumab or bevacizumab injections over the time may demonstrate tachyphylaxis or a diminished therapeutic response to those agents [32]. The mechanisms underlying this phenomenon are thought to be multifactorial, involving, for example, upregulation of VEGF from local macrophages, altered expression of surface receptors, and humoral responses [32].

Some studies have demonstrated anatomical and visual improvement with the switch to ranibizumab or aflibercept in patients with persistent DME treated previously with bevacizumab $[30,31,33]$. Ciulla et al. [30] reported in their retrospective study that ranibizumab was useful in eyes with persistent DME treated previously with macular laser photocoagulation, bevacizumab, and triamcinolone, with statistically significant improvement in CRT from 384 to $335 \mu \mathrm{m}$ and visual acuity improvement from 20/100 to 20/90 after an average of 7 injections over 48 weeks [30, 31, 33]. Lack of group control, small sample size, and retrospective design limit interpretation from many of these studies.

\section{Corticosteroids}

Corticosteroids have several mechanisms of action that include decrease in VEGF synthesis, in leukocyte migration, and in several proinflammatory cytokines such as IL- 6 and IL-8; enhancement of the barrier function of vascular endothelial cell tight junctions; and downregulation of ICAM-1 expression [4, 34]. Currently, corticosteroid agents serve as the second-line drug for DME treatment and specifically as the main therapy in control of persistent DME. Cataract formation and glaucoma are known side effects of intravitreal corticosteroids [4, 34]. Intravitreal triamcinolone, fluocinolone insert, and dexamethasone implant are agents available for DME treatment.

Triamcinolone inhibits the arachidonic acid pathway, thereby inhibiting production of the prostaglandins that 
trigger the inflammatory response. The Protocol B from the DRCR.net showed that over a 2-year period, focal/ grid photocoagulation is more effective and has fewer side effects than triamcinolone for patients with DME [35]. Jeon and Lee [35] have demonstrated that intravitreal triamcinolone had beneficial effects on DME unresponsive to anti-VEGF agents in improved visual acuity and reduced CRT. However, these effects were not sustained for $>3$ months [35].

Dexamethasone implant is a biodegradable polymer that gradually releases low-dose dexamethasone for several months after being injected into the vitreous cavity, maintaining drug concentration for 3-6 months. The peak concentration of the drug has been reached in the vitreous at the second month, and the highest efficacy of the implant was shown to occur between the first and third month [34, 36, 37]. The MEAD study showed robust long-term improvement in vision and macular thickness in patients with DME treated with dexamethasone implant, with a mean of only 4-5 injections over 3 years [37]. Jung and Lee [38] have demonstrated that vitrectomy combined with intraoperative dexamethasone implant may produce a clinically meaningful and statistically significant benefit in the treatment of persistent DME. Some studies showed that the dexamethasone implant provides a more significant improvement in CRT compared with aflibercept in treatment-naïve DME with SRF, a marked inflammatory phenotype, with a low number of injections as an advantage [39].

Fluocinolone intravitreal implant is a small, nonbiodegradable implant and applied as an injection using a 25 -gauge injector via pars plana into the vitreous cavity, releasing an average of $0.2 \mu \mathrm{g} /$ day of fluocinolone acetonide for up to 36 months [40]. The FAME study showed a substantial visual benefit for up to 3 years in patients with persistent DME, and fluocinolone provides an option for patients who do not respond to other therapy [40].

The multifactorial pathogenesis of DME provides rationale for the combination therapies [4]. Combining bevacizumab with dexamethasone implant was found to achieve a greater reduction on CRT, but achieved a similar visual acuity gain compared to bevacizumab alone in patients with persistent DME [4]. Shah and Maturi [4] have demonstrated that in eyes with persistent DME, after multiple anti-VEGF injections, the addition of dexamethasone implant with continued ranibizumab injections produced no better improvement of visual acuity at 6 months than the monotherapy with ranibizumab, even though there was a significantly greater reduction in CRT in the combination group $[34,41]$.

New Insights in Resistant Diabetic Macular Edema

\section{Laser}

Macular laser photocoagulation has been in use for the last several decades to treat DME since the ETDRS report was published in 1985 [42]. Currently, however, antiVEGF therapy has replaced laser as the first-line treatment. The main disadvantage of anti-VEGF monotherapy is the high frequency of injections per year and the risk of injection-associated adverse events, such as endophthalmitis. Patients may require as many as 8 injections per year, and this high frequency places a huge economic burden on patients and healthcare systems [43]. Macular laser photocoagulation may cause macular scars that result in central scotoma, and it can also be complicated by choroidal neovascularization and subretinal fibrosis. To prevent these from occurring, subthreshold laser has been developed to deliver laser energy below the threshold of causing permanent tissue damage, and it has shown to alter metabolic activity, RPE gene expression, and enhance the expression of heat shock proteins [44]. Subthreshold laser uses longer wavelengths and delivers energy in a micropulse fashion [42, 43, 45].

Micropulse laser has emerged as the main modality to achieve subthreshold treatment by decreasing the "duty cycle" of the laser. Instead of one single continuous pulse, the laser is divided into numerous short repetitive pulses, within 100-300 ms "on" cycle and 1,700-1,900 ms "off" [46]. This effectively decreases the "duty cycle" of the laser as low as $5-10 \%$ of the conventional laser [42]. Micropulse laser has been shown in several randomized clinical trials to be as effective as conventional laser in the DME treatment [42]. Chen et al. [46] have showed that the micropulse diode laser treatment resulted in better visual acuity compared to focal/grid macular laser. However, the 2 types of treatment seem to have similar anatomical outcome. Some authors have described the association between anti-VEGF therapy and micropulse laser with improvement of visual and anatomical outcome, with a significant decrease in the number of additional injections required. These studies have limitations such as a small sample size, retrospective design, and short followup $[43,46]$.

Alternatively, in 2005, Topcon developed a pattern scanning laser system capable of applying multiple laser spots in a short period of time [44]. PASCAL streamline yellow with endpoint management software allows grid pattern photocoagulation and the ability to calculate the level of subthreshold energy required [44]. Hamada et al. [44] have demonstrated 10 cases of patients with DME treated with subthreshold laser using endpoint management with a significant decrease of the CRT, while there

Ophthalmologica 2021;244:485-494 489 
were no significant changes in BCVA. Further clinical trials are needed to corroborate these findings.

Microaneurysms are the hallmark of DR and DME; however, the spectrum of microvascular abnormalities is far more complex, comprising large microvascular abnormalities, which can reach the diameter of several micra $(150 \mu \mathrm{m}$ or more) and receive the term of telangiectatic capillaries (TC). The TC are better seen by indocyanine green angiography than by fluorescein angiography, showing delayed and prolonged indocyanine green angiography staining. Some authors have reported anatomical and visual improvement of DME after target photocoagulation of the TC [47].

\section{Surgery}

Pars plana vitrectomy (PPV) with or without internal limiting membrane (ILM) peeling has been described for persistent DME treatment [48]. Consistent with the guidelines for the management of DME by retinal specialists, PPV is currently recommended as a therapeutic option in cases of DME associated with tractional changes in the vitreomacular interface. In the absence of vitreomacular traction, there is no consensus on the role of PPV [48].

PPV is thought to play a role in nontractional cases, allowing VEGF and other proinflammatory cytokines to diffuse away from the macula more easily and increasing the oxygen supply in ischemic areas. On the other hand, postvitrectomy eyes have a decreased viscosity of the vitreous cavity, interfering with intraocular pharmacokinetics and reducing the half-life of intravitreal agents $[4,49]$. Jackson et al. [49] in their meta-analysis showed that PPV produced both structural and functional benefit in eyes with DME, but the functional benefit was not significantly better than that obtained using macular laser. Nakajima et al. [50] in their meta-analysis demonstrated similar visual acuity outcomes using PPV with ILM peeling versus no ILM peeling in patients with DME.

\section{Future Perspectives}

Anti-VEGF therapy continues as the first-line treatment for CI-DME. The main disadvantage of this therapy is the high frequency of injections. Patients may require as many as 8 injections per year, and this focuses a huge economic burden on patients and healthcare systems [43]. Given the burden of frequent injections, agents with longer duration of action are being investigated intensively [4]. This review focuses on new anti-VEFG agents that are in advanced stage of clinical development: conbercept, brolucizumab, and faricimab.

\section{Conbercept}

Conbercept is a recombinant fusion protein composed of the second Ig domain of VEGFR-1 and the third and fourth Ig domains of VEGFR-2 and the constant region $(\mathrm{Fc})$ of human IgG $[26,51]$. The structure of conbercept differs from aflibercept in that it incorporates the fourth extracellular domain of VEGFR-2, which was demonstrated to stabilize the receptor-ligand complex and further extend the half-life of conbercept $[26,51]$. It is designed as a receptor decoy with high affinity for all VEGF isoforms and PIGF $[26,51]$. The inhibitory effects of conbercept have been evaluated in vitro and in vivo, indicating that conbercept exerts potent antiangiogenic effects [52]. The PHOENIX study evaluated the efficacy and safety of intravitreal injections of $0.5 \mathrm{mg}$ conbercept for the treatment of neovascular age-related macular degeneration (AMD) and showed significant visual and anatomic benefits with 3 initial monthly doses of conbercept (loading dose) followed by quarterly dosing of conbercept $[26,51,52]$.

\section{Brolucizumab}

Brolucizumab is a low-molecular-weight humanized single-chain variable $(\mathrm{scFc}$ ) antibody fragment VEGF inhibitor [53]. It binds and inhibits the 3 major isoforms of VEGF-A (VEGF110, VEGF121, and VEGF165) and prevents interaction with receptors VEGF-1 and VEGF-2 [53]. Brolucizumab is approved in the USA for the treatment of neovascular AMD based on the HAWK and HARRIER studies [53]. These studies have reported that brolucizumab provides comparable vision gains to aflibercept, with greater retinal fluid resolution [53]. The recommended regimen dosage is $6 \mathrm{mg}$ monthly for the first 3 months and then once every 8-12 weeks [54]. Baumal et al. [55] have reported a case series of 15 eyes from 10 practices in the USA that demonstrated retinal vasculitis and intraocular inflammation within the first 3 months of its availability.

\section{Faricimab}

DME is multifactorial and involves other angiogenic factors and inflammatory cytokines that are not addressed with anti-VEGF therapy [56, 57]. Angiopoietin (Ang) pathway regulates the vascular homeostasis and vascular permeability [56, 57]. Upregulation of Ang-2 was noted in conditions such as hypoxia, hyperglycemia, and oxidative stress [56,57]. Faricimab, a novel anti- 
Ang-2/anti-VEGF bispecific antibody designed for intraocular use, has been produced to inhibit these 2 pathways $[56,57]$. The phase 2 BOULEVARD study reported that $6 \mathrm{mg}$ intravitreal faricimab given monthly for 20 weeks had superior gains in visual acuity compared with ranibizumab in treatment-naïve patients with $\operatorname{DME}[56,57]$.

\section{Port Delivery System}

The port delivery system (PDS) with ranibizumab is a novel, innovative, long-acting drug delivery system that enables continuous delivery of a customized formulation of ranibizumab into the vitreous $[58,59]$. The PDS includes a permanent, refillable implant that is surgically inserted through a small incision in the sclera and pars plana $[58,59]$. Ranibizumab moves by passive diffusion down a concentration gradient from the implant reservoir, through a porous metal release control element specifically designed for ranibizumab, and into the vitreous cavity $[58,59]$. The PDS implantation procedure is well tolerated, self-retaining, and performed in a sutureless fashion, and the refill procedure is office based $[58,59]$. The phase 2 LADDER clinical trial showed visual and anatomic outcomes comparable with monthly intravitreal ranibizumab. The mean refill time was 15 months, with $80 \%$ of the patients not requiring a refill per protocol for the first 6 months $[58,59]$.

\section{Abicipar Pegol}

Ankyrin repeats are one of the most common protein patterns in nature, and they form ankyrin repeat proteins when stacked together [2-4]. Libraries of artificially stacked designed ankyrin repeat proteins (DARPin) are a novel class of proteins that demonstrate high affinity and specificity for binding to specific proteins. Abicipar pegol is a specifically designed DARPin that binds and inhibits VEGF and when administered at a dose of $0.4 \mathrm{mg}$ intravitreal, VEGF inhibition was observed for 8-12 weeks in the aqueous humor [2-4]. However, there are concerns regarding an increased incidence of intraocular inflammation in eyes treated with Abicipar pegol [2-4]. The phase 2 PALM study was conducted on 151 patients with DME and reported visual acuity improvement with Abicipar pegol [2-4].

\section{Interleukin Inhibitors and Chemokine Inhibitors}

$\mathrm{DM}$ is a systemic disease, and it has been associated with higher serum levels of several cytokines, chemokines, and angiogenic factors. In this context, novel systemically administered therapeutic agents are in development to treat DR and DME $[2,3,60]$. Recent studies have shown that levels of insulin-like growth factor, inflammatory cytokines such as IL- 1 and IL-6, and chemokines such as monocyte chemoattractant protein are increased in the vitreous of patients with DME $[2,3,60]$. Pharmacological inhibition of these factors appears to be a promising approach for treating DME $[2,3,60]$. The use of the IL-1 receptor antagonist has been shown to significantly reduce glucose-induced abnormalities in the bovine retina. R07200220, a recombinant fully humanized immunoglobulin G2 (IgG2) isotype monoclonal antibody that potently binds IL- 6 and inhibits all forms of IL- 6 signaling, is intended for the treatment of DME, uveitic macular edema, and neovascular AMD [2, 3, 60]. Further clinical studies involving inhibition of these pathways may elucidate whether there are beneficial effects for those therapeutic strategy for DME $[2,3,60]$.

\section{Rho-Kinase Inhibitors}

Rho-associated protein kinase activity is upregulated in diabetic eyes and plays an important role in the pathogenesis of microvascular complication in DR/DME [2-4, 61]. Rho-associated protein kinase inhibitors may be used to enhance the efficacy of anti-VEGF in treating persistent DME $[2-4,61]$. AR-13403 (Aeri Pharmaceuticals) is a sustained-release intravitreal implant designed to treat neovascular AMD and DME that inhibits both Rho-kinase and protein kinase $C[2-4,61]$.

\section{Neuroprotective Agents}

Neurodegeneration is an early event in the pathogenesis of DR and DME; therefore, it is critical to develop agents that can target the disease process in early stages $[2-4,62]$. Retinal neuroprotective drugs such as topical brimonidine and somatostatin have been assessed in the EUROCONDOR study $[2-4,62]$. This study reported that topical treatment with these agents did not seem to be useful in preventing the development of neurodegeneration; however, these protective agents were reported to be effective in preventing the progression of neurodegeneration in patients that already had some degree of neurodegeneration $[2-4,62]$.

\section{Conclusion}

Controlling hyperglycemia is critical to minimizing the risk of onset and progression of DME. The American Diabetes Association recommends glycemic control targeting a hemoglobin $\mathrm{A} 1 \mathrm{C}$ of $7.0 \%$ or lower for most people with DM [2]. Currently, the mainstay of the DME 
treatment includes anti-VEGF agents, corticosteroids, and laser therapy. The introduction of anti-VEGF has been a paradigm shift in the DME management, with substantial improvement in visual outcome for patients with DME. However, this treatment implies in a high economic burden due to the need of multiple intravitreal injections.

Despite the improvement in DME treatment with anti-VEGF therapy, persistent DME remains a challenge due to the extremely complex pathogenesis and the involvement of several different biochemical pathways. Upregulation of VEGF, Ang-2, IL-6, IL-8, PGF, ICAM-1, pigment epithelium-derived factor, and other cytokines has been described to play an important role in DME pathogenesis. Recently, there is emerging evidence suggesting that retinal neurodegeneration is an early event in $\mathrm{DR} / \mathrm{DME}$. Therefore, it is critical to identify each contributing component to plan a personalized treatment for DME.

Future treatment perspectives include an individualized management of DME, with the development of novel anti-VEGF agents with longer duration of action in the vitreous cavity, PDS for anti-VEGF, serum and aqueous humor measurement of inflammatory cytokines, evaluation of OCT biomarkers to guide therapy, agents target- ing different biochemical pathways such as angiopoetin-2 and IL-6, and neuroprotective agents. These new targets may potentially serve as an alternative therapy or be used in combination with anti-VEGF. Multicenter randomized clinical trials may provide level 1 evidence for these novel agents in the future.

\section{Conflict of Interest Statement}

The authors declare that they have no conflicts of interest.

\section{Funding Sources}

The authors did not receive any funding for this study.

\section{Author Contributions}

Dante A.K. Kuroiwa: conceptualization; data acquisition; project administration; and writing - original draft, review, and editing. Fernando K. Malerbi: conceptualization; data acquisition; project administration; and writing - review and editing. Caio V.S. Regatieri: conceptualization; data acquisition; project administration; supervision; and writing - review and editing.

\section{References}

1 Das A, McGuire PG, Rangasamy S. Diabetic macular edema: pathophysiology and novel therapeutic targets. Ophthalmology. 2015; 122(7):1375-94.

2 Agarwal A, Afridi R, Hassan M, Sadiq MA, Sepah YJ, Do DV, et al. Novel therapies in development for diabetic macular edema. Curr Diab Rep. 2015;15(10):75.

3 Sadiq MA, Halim MS, Hassan M, Onghanseng N, Karaca I, Agarwal A, et al. Pharmacological agents in development for diabetic macular edema. Int J Retina Vitreous. 2020; 6(1):29-11.

4 Shah SU, Maturi RK. Therapeutic options in refractory diabetic macular oedema. Drugs. 2017;77(5):481-92.

5 Felfeli T, Juncal VR, Hillier RJ, Mak MYK, Wong DT, Berger AR, et al. Aqueous humor cytokines and long-term response to anti-vascular endothelial growth factor therapy in diabetic macular edema. Am J Ophthalmol. 2019;206:176-83.

6 Harding JL, Pavkov ME, Magliano DJ, Shaw JE, Gregg EW. Global trends in diabetes complications: a review of current evidence. Diabetologia. 2019;62(1):3-16.
7 Ogurtsova K, da Rocha Fernandes JD, Huang Y, Linnenkamp U, Guariguata L, Cho NH, et al. IDF diabetes atlas: global estimates for the prevalence of diabetes for 2015 and 2040. Diabetes Res Clin Pract. 2017;128:40-50.

8 Ting DS, Cheung GC, Wong TY. Diabetic retinopathy: global prevalence, major risk factors, screening practices and public health challenges: a review. Clin Exp Ophthalmol. 2016;44(4):260-77.

9 Varma R, Bressler NM, Doan QV, Gleeson M, Danese M, Bower JK, et al. Prevalence of and risk factors for diabetic macular edema in the United States. JAMA Ophthalmol. 2014; 132(11):1334-40.

10 Lee R, Wong TY, Sabanayagam C. Epidemiology of diabetic retinopathy, diabetic macular edema and related vision loss. Eye Vis. 2015; 2(1):17-25.

11 Daruich A, Matet A, Moulin A, Kowalczuk L, Nicolas M, Sellam A, et al. Mechanisms of macular edema: beyond the surface. Prog Retin Eye Res. 2018;63:20-68.

12 Kwon JW, Jee D. Aqueous humor cytokine levels in patients with diabetic macular edema refractory to anti-VEGF treatment. PLoS One. 2018;13(9):e0203408-9.
13 Aiello LM, Ferris FL. Photocoagulation for diabetic macular edema. Arch Ophthalmol. 1987;105(9):1163.

14 Browning DJ, Altaweel MM, Bressler NM, Bressler SB, Scott IU. Diabetic macular edema: what is focal and what is diffuse? Am J Ophthalmol. 2008;146(5):649.

15 Parravano M, Costanzo E, Querques G. Profile of non-responder and late responder patients treated for diabetic macular edema: systemic and ocular factors. Acta Diabetol. 2020; 57(8):911-21.

16 Bressler SB, Ayala AR, Bressler NM, Melia M, Qin H, Ferris FL, et al. Persistent macular thickening after ranibizumab treatment for diabetic macular edema with vision impairment. JAMA Ophthalmol. 2016;134(3):27885.

17 Busch C, Zur D, Fraser-Bell S, Laíns I, Santos AR, Lupidi M, et al. Shall we stay, or shall we switch? Continued anti-VEGF therapy versus early switch to dexamethasone implant in refractory diabetic macular edema. Acta Diabetol. 2018;55(8):789-96. 
18 Santos AR, Alves D, Santos T, Figueira J, Silva R, Cunha-Vaz JG. Measurements of retinal fluid by optical coherence tomography leakage in diabetic macular edema: a biomarker of visual acuity response to treatment. Retina. 2019;39(1):52-60.

19 Staurenghi G, Sadda S, Chakravarthy U, Spaide RF. Proposed lexicon for anatomic landmarks in normal posterior segment spectral-domain optical coherence tomography: the IN OCT consensus. Ophthalmology. 2014;121(8):1572-8.

20 Hsieh YT, Alam MN, Le D, Hsiao CC, Yang $\mathrm{CH}$, Chao DL, et al. OCT angiography biomarkers for predicting visual outcomes after ranibizumab treatment for diabetic macular edema. Ophthalmol Retina. 2019;3(10):82634.

21 Sun JK, Lin MM, Lammer J, Prager S, Sarangi $\mathrm{R}$, Silva PS, et al. Disorganization of the retinal inner layers as a predictor of visual acuity in eyes with center-involved diabetic macular edema. JAMA Ophthalmol. 2014;132(11): 1309-16.

22 Rayess N, Rahimy E, Ying GS, Bagheri N, Ho $\mathrm{AC}$, Regillo $\mathrm{CD}$, et al. Baseline choroidal thickness as a predictor for response to antivascular endothelial growth factor therapy in diabetic macular edema. Am J Ophthalmol. 2015;159(1):85-3.

23 Vujosevic S, Bini S, Torresin T, Berton M, Midena G, Parrozzani R, et al. Hyperreflective retinal spots in normal and diabetic eyes: Bscan and en face spectral domain optical coherence tomography evaluation. Retina. 2017;37(6):1092-103.

24 Zur D, Iglicki M, Busch C, Invernizzi A, Mariussi M, Loewenstein A, et al. OCT biomarkers as functional outcome predictors in diabetic macular edema treated with dexamethasone implant. Ophthalmology. 2018;125(2): $267-75$.

25 Hillier RJ, Ojaimi E, Wong DT, Mak MYK, Berger AR, Kohly RP, et al. Aqueous humor cytokine levels and anatomic response to intravitreal ranibizumab in diabetic macular edema. JAMA Ophthalmol. 2018;136(4):3828.

26 Cai S, Yang Q, Li X, Zhang Y. The efficacy and safety of aflibercept and conbercept in diabetic macular edema. Drug Des Devel Ther. 2018; 12:3471-83.

27 Nguyen QD, Brown DM, Marcus DM, Boyer DS, Patel S, Feiner L, et al. Ranibizumab for diabetic macular edema: results from 2 phase iii randomized trials: RISE and RIDE. Ophthalmology. 2012;119(4):789-801.

28 Heier JS, Bressler NM, Avery RL, Bakri SJ, Boyer DS, Brown DM, et al. Comparison of aflibercept, bevacizumab, and ranibizumab for treatment of diabetic macular edema: extrapolation of data to clinical practice. JAMA Ophthalmol. 2016;134(1):95-9.
29 Elman MJ, Elman MJ, Aiello LP, Beck RW, Bressler NM, Bressler SB, et al. Randomized trial evaluating ranibizumab plus prompt or deferred laser or triamcinolone plus prompt laser for diabetic macular edema. Ophthalmology. 2010;117(6):1064-77.e35.

30 Ciulla TA, Hussain RM, Ciulla LM, Sink B, Harris A. Ranibizumab for diabetic macular edema refractory to multiple prior treatments. Retina. 2016;36(7):1292-7.

31 Moradi A, Sepah YJ, Sadiq MA, Nasir H, Kherani S, Sophie R, et al. Vascular endothelial growth factor trap-eye (Aflibercept) for the management of diabetic macular edema. World J Diabetes. 2013;4(6):303.

32 Rahimy E, Shahlaee A, Khan MA, Ying GS, Maguire JI, Ho AC, et al. Conversion to aflibercept after prior anti-VEGF therapy for persistent diabetic macular edema. Am J Ophthalmol. 2016;164:118-27.e2.

33 Bahrami B, Hong T, Schlub TE, Chang AA Aflibercept for persistent diabetic macular edema: forty-eight-week outcomes. Retina. 2019;39(1):61-8.

34 Călugăru D, Călugăru M. Aflibercept in diabetic macular edema refractory to previous bevacizumab: outcomes and predictors of success. Graefes Arch Clin Exp Ophthalmol. 2018;256(7):1353-4

35 Jeon S, Lee WK. Effect of intravitreal triamcinolone in diabetic macular edema unresponsive to intravitreal bevacizumab. Retina. 2014;34(8):1606-11.

36 Khan Z, Kuriakose RK, Khan M, Chin EK, Almeida DRP. Effcacy of the intravitreal sustained-release dexamethasone implant for diabetic macular edema refractory to antivascular endothelial growth factor therapy: meta-analysis and clinical implications. Ophthalmic Surg Lasers Imaging Retin. 2017; 48(2):160-6.

37 Boyer DS, Yoon YH, Belfort R, Bandello F, Maturi RK, Augustin AJ, et al. Three-year, randomized, sham-controlled trial of dexamethasone intravitreal implant in patients with diabetic macular edema. Ophthalmology. 2014;121(10):1904-14.

38 Jung YH, Lee Y. Efficacy of vitrectomy combined with an intraoperative dexamethasone implant in refractory diabetic macular edema. Acta Diabetol. 2019;56(6):691-6.

39 Ozsaygili C, Duru N. Comparison of intravitreal dexamethasone implant and aflibercept in patients with treatment-naive diabetic macular edema with serous retinal detachment. Retina. 2020;40(6):1044-52.

40 Massa H, Nagar AM, Vergados A, Dadoukis $\mathrm{P}$, Patra S, Panos GD. Intravitreal fluocinolone acetonide implant (ILUVIEN ${ }^{\circledR}$ ) for diabetic macular oedema: a literature review. J Int Med Res. 2019;47(1):31-43.

41 Mehta H, Hennings C, Gillies MC, Nguyen V, Campain A, Fraser-Bell S. Anti-vascular endothelial growth factor combined with intravitreal steroids for diabetic macular oedema. Cochrane Database Syst Rev. 2018;4(4): CD011599.
42 Su D, Hubschman JP. A review of subthreshold micropulse laser and recent advances in retinal laser technology. Ophthalmol Ther. 2017;6(1):1-6.

43 Inagaki K, Hamada M, Ohkoshi K. Minimally invasive laser treatment combined with intravitreal injection of anti-vascular endothelial growth factor for diabetic macular oedema. Sci Rep. 2019;9(1):7585-8.

44 Hamada M, Ohkoshi K, Inagaki K, Ebihara N, Murakami A. Subthreshold photocoagulation using endpoint management in the PASCAL system for diffuse diabetic macular edema. J Ophthalmol. 2018;2018:1-9.

45 Moisseiev E, Abbassi S, Thinda S, Yoon J, Yiu G, Morse LS. Subthreshold micropulse laser reduces anti-VEGF injection burden in patients with diabetic macular edema. Eur J Ophthalmol. 2018;28(1):68-73.

46 Chen G, Tzekov R, Li W, Jiang F, Mao S, Tong Y. Subthreshold micropulse diode laser versus conventional laser photocoagulation for diabetic macular edema: a meta-analysis of randomized controlled trials. Retina. 2016; 36(11):2059-65.

47 Castro Farías D, Matsui Serrano R, Bianchi Gancharov J, De Dios Cuadras U, Sahel J, Graue Wiechers $\mathrm{F}$, et al. Indocyanine green angiography for identifying telangiectatic capillaries in diabetic macular oedema. $\mathrm{Br} \mathrm{J}$ Ophthalmol. 2020;104(4):509-13.

48 Hagenau F, Vogt D, Ziada J, Guenther SR, Haritoglou C, Wolf A, et al. Vitrectomy for diabetic macular edema: optical coherence tomography criteria and pathology of the vitreomacular interface. Am J Ophthalmol. 2019;200:34-46.

49 Jackson TL, Nicod E, Angelis A, Grimaccia F, Pringle E, Kanavos P. Pars plana vitrectomy for diabetic macular edema: a systematic review, meta-analysis, and synthesis of safety literature. Retina. 2017;37(5):886-95.

50 Nakajima T, Roggia MF, Noda Y, Ueta T. Effect of internal limiting membrane peeling during vitrectomy for diabetic macular edema: systematic review and meta-analysis. Retina. 2015;35(9):1719-25.

51 Liu K, Song Y, Xu G, Ye J, Wu Z, Liu X, et al Conbercept for treatment of neovascular agerelated macular degeneration: results of the randomized phase 3 PHOENIX Study. Am J Ophthalmol. 2019;197:156-67.

52 Liu XY, Peng XY, Wang S, You QS, Li YB, Xiao YY, et al. Features of optical coherence tomography for the diagnosis of vogt-koyanagi-harada disease. Retina. 2016;36(11): 2116-23.

53 Dugel PU, Singh RP, Koh A, Ogura Y, Weissgerber G, Gedif K, et al. HAWK and HARRIER: ninety-six-week outcomes from the phase 3 trials of brolucizumab for neovascular age-related macular degeneration. Ophthalmology. 2021;128(1):89-99.
New Insights in Resistant Diabetic Macular Edema
Ophthalmologica 2021;244:485-494 DOI: 10.1159/000516614 
54 Markham A. Brolucizumab: first approval. Drugs. 2019;79(18):1997-2000.

55 Baumal CR, Spaide RF, Vajzovic L, Freund $\mathrm{KB}$, Walter SD, John V, et al. Retinal vasculitis and intraocular inflammation after intravitreal injection of brolucizumab. Ophthalmology. 2020;127(10):1345-59.

56 Sharma A, Kumar N, Kuppermann BD, Bandello F, Loewenstein A. Faricimab: expanding horizon beyond VEGF. Eye. 2020;34(5):802-4.

57 Sahni J, Patel SS, Dugel PU, Khanani AM, Jhaveri CD, Wykoff CC, et al. Simultaneous inhibition of angiopoietin-2 and vascular endothelial growth factor-A with faricimab in diabetic macular edema: BOULEVARD phase 2 randomized trial. Ophthalmology. 2019;126(8):1155-70.
58 Sharma A, Kumar N, Parachuri N, Kuppermann BD, Bandello F, Regillo CD. Ranibizumab port delivery system (RPDS): realising long awaited dream of prolonged VEGF suppression. Eye. 2020;34(3):422-3.

59 Sharma A, Kumar N, Kuppermann BD, Francesco B. Re: Campochiaro et al.: the port delivery system with ranibizumab for neovascular age-related macular degeneration: results from the randomized phase 2 Ladder clinical trial (Ophthalmology. 2019;126:1141-1154). Ophthalmology. 2019;126(11):e87-8.
60 Figueras-Roca M, Molins B, Sala-Puigdollers A, Matas J, Vinagre I, Ríos J, et al. Peripheral blood metabolic and inflammatory factors as biomarkers to ocular findings in diabetic macular edema. PLoS One. 2017;12(3): e0173865.

61 Minami Y, Song YS, Ishibazawa A, Omae T, Ro-mase T, ishiko $S$, et al. Effect of ripasudil on diabetic macular edema. Sci Rep. 2019; 9(1):3703-9.

62 Simó R, Hernández C. Neurodegeneration in the diabetic eye: new insights and therapeutic perspectives. Trends Endocrinol Metab. 2014; $25(1): 23-33$ 